\title{
Imatinib mesylate (STI571) enhances amrubicin-induced cytotoxic activity through inhibition of the phosphatidylinositol 3-kinase/Akt pathway in small cell lung cancer cells
}

\author{
HISASHI SUYAMA, TADASHI IGISHI, YASUTO UEDA, YASUSHI SHIGEOKA, \\ MASAHIRO KODANI, MASATO MORITA, KENICHI TAKEDA, TAKASHI SUMIKAWA, \\ HIROFUMI NAKAZAKI, KEIJI MATSUNAMI, SHINGO MATSUMOTO and EIJI SHIMIZU
}

Division of Medical Oncology and Molecular Respirology, Faculty of Medicine, Tottori University, Yonago 683-8504, Japan

Received July 30, 2009; Accepted September 17, 2009

DOI: 10.3892/or_00000626

\begin{abstract}
Small cell lung cancer (SCLC) is characterized by autocrine mechanisms. Stem cell factor (SCF) and its receptor c-kit can activate Akt and extracellular signal-regulated kinase (Erk) pathways. Imatinib mesylate (STI571) can inhibit c-kit tyrosine kinase activity, but clinical trials have resulted in failure. We investigated the possibility of SCF/c-kit-targeted therapy against SCLC. Using c-kit-positive SCLC cells (H209 and H69 cells) and SCF as a model of the autocrine mechanisms, the effects of SCF, LY294002, PD98059 or STI571 on Akt and Erk were assessed by Western blot analysis. The cell growth inhibitions of cisplatin, etoposide irinotecan and amrubicin (AMR) with or without SCF, LY294002, PD98059 or STI571 were evaluated by MTT assay. Treatment with SCF activated Akt and Erk and the activations were inhibited by STI571 in H209 but not in H69 cells. LY294002 and PD98059 inhibited SCF-induced Akt and Erk activation in H209 cells, respectively. STI571 alone did not exert growth inhibition in the SCF-treated cells. In H209 cells, SCF decreased the cytotoxicity of AMR, but not of other drugs. In H69 cells, SCF did not affect sensitivity to any drugs. LY294002 but not PD98059 restored or enhanced AMR-sensitivity in SCF-treated H209 or untreated H69 cells, respectively. STI571 restored the AMR-sensitivity of SCF-treated H209 cells to the basal level. If the SCF/c-kit contributes to Akt activation in vivo, the combination of STI571 and AMR may be effective against SCLC. Additionally, using a combination of AKT inhibitors and AMR may be a promising treatment in the future.
\end{abstract}

Correspondence to: Dr Tadashi Igishi, Division of Medical Oncology and Molecular Respirology, Faculty of Medicine, Tottori University, Yonago 683-8504, Japan

E-mail: igishi@med.tottori-u.ac.jp

Key words: small cell lung cancer, amrubicin, imatinib mesylate, STI571, Akt

\section{Introduction}

Small cell lung cancer (SCLC) is a highly aggressive neoplasm characterized by a high growth fraction, short doubling time and high rate of metastasis (1), but is sensitive to chemotherapy (2). Systemic chemotherapy prolongs the survival of SCLC patients (3), and cisplatin (CDDP), etoposide (VP-16), topoisomerase I inhibitors [irinotecan (CPT-11) and topotecan] $(4,5)$ and amrubicin (AMR) (6) (a totally synthetic 9-aminoanthracycline) are used in regular clinical practice in Japan. Among these chemotherapeutic agents, the combination chemotherapy of CDDP and VP-16 is considered the standard first line chemotherapeutic regimen worldwide (7). Despite high response rates with these first line CDDP-based chemotherapies, most patients eventually experience disease progression. Accordingly, novel chemotherapeutic regimens based on new conceptions are needed for treating SCLC patients.

SCLC is, per se, characterized by several autocrine growth mechanisms including stem cell factor (SCF) and its receptor c-kit, and c-kit overexpression is found in up to $70 \%$ of SCLC (8). In view of c-kit signal transduction, an SCF/c-kit can activate several intracellular pathways including phosphatidylinositol 3-kinase (PI3K)/Akt and mitogen-activated protein kinase kinase/extracellular signal-regulated kinase (MEK/Erk) pathways (9).

Imatinib mesylate (STI571) was developed as a Bcr-Abl inhibitor and has become a standard therapeutic drug for chronic myelogenous leukemia (10). STI571 can also inhibit c-kit tyrosine kinase activity and has high efficacy in chemoresistant c-kit-positive gastrointestinal stromal tumors (11).

Taking these previous findings into consideration, researchers have evaluated the efficacy of STI571 in the treatment of SCLC. Two phase II studies of STI571 alone in unselected (12) or c-kit-positive (13) SCLC patients failed to show its efficacy. Furthermore, a phase I clinical trial using STI571, CDDP and CPT-11 revealed no combination effects between STI571 and these cytotoxic agents (14).

However, if the autocrine mechanism of SCF is functioning in SCLC in vivo, it is still possible that the SCF/c-kit-targeted therapy may be effective in the treatment of SCLC. Thus, we 
investigated the effect of SCF and STI571 on SCLC cell growth and the combination effects of STI571 with other chemotherapeutic agents used in regular clinical practice using c-kit-positive SCLC cells.

\section{Materials and methods}

Chemicals and reagents. CDDP (a gift from Nippon Kayaku, Co. Ltd, Tokyo, Japan) and AMR (a gift from Dainippon Sumitomo Pharma Co. Ltd, Osaka, Japan) were dissolved in distilled water and stored at $-20^{\circ} \mathrm{C}$ after filtration. VP-16 (Wako Pure Chemical Industries, Ltd, Osaka, Japan) and CPT-11 (a gift from Daiichi Pharmaceutical, Co. Ltd., Tokyo, Japan) were dissolved in dimethylsulfoxide and stored at $-20^{\circ} \mathrm{C}$. Recombinant human SCF (a gift from Kirin Brewery Co. Ltd, Tokyo, Japan) was stored at $4^{\circ} \mathrm{C}$. 2'-Amino-3'methoxyflavone (PD98059) (Calbiochem, San Diego, CA, USA) and 2-(4-Morpholinyl)-8-phenyl-4H-1-benzopyran-4one (LY294002) (Sigma-Aldrich Japan, Tokyo, Japan) was dissolved in dimethylsulfoxide and stored at $-20^{\circ} \mathrm{C}$. 3- $(4,5-$ dimethylthiazol-2-yl)-2,5-diphenyltetrazolium bromide (MTT) (Sigma Chemical Co., St. Louis, MO, USA) was dissolved in phosphate-buffered saline (PBS) and stored at $-20^{\circ} \mathrm{C}$.

Cells. H209 and H69 human SCLC cell lines were provided by Dr A.F. Gazdar and Dr H. Oie (NCI-Navy Medical Oncology Branch, NIH, Bethesda, USA). The cells were maintained in Dulbecco's modified Eagle's medium (DMEM) supplemented with $10 \%$ fetal calf serum (FCS) and antibiotics. The cells were grown in a humidified atmosphere of $5 \% \mathrm{CO}_{2}-95 \%$ air.

MTT assay. The effects of SCF on the cell proliferation in the H209 and H69 cells were measured by MTT assay using 96-well flat bottom multiplates (Nalge Nunc International K.K., Denmark). The cells were counted with a hematocytometer and $5 \times 10^{4}$ cells were treated with or without $100 \mathrm{ng} / \mathrm{ml}$ of SCF for 24, 48 or $72 \mathrm{~h}$. Next, $20 \mu \mathrm{g}$ of MTT in $10 \mu \mathrm{l}$ PBS was added to each well and incubation was performed for an additional $4 \mathrm{~h}$. Thereafter, $100 \mu \mathrm{l}$ of $0.04 \mathrm{~N} \mathrm{HCl}$ in 2-propanol was added and incubated overnight in order to solubilize the MTT formazan crystals. The absorbance of each well was measured at a 570-nm wavelength (reference $650 \mathrm{~nm}$ ) using a scanning multiwell spectrophotometer (MPR A4i, Tosoh Co, Tokyo, Japan). The cytotoxic activity of AMR in the H209 and H69 cells was evaluated by MTT assay in the same way as described above using $100 \mathrm{ng} / \mathrm{ml}$ of SCF and $10 \mu \mathrm{M}$ of STI571.

Cell lysis, immunoprecipitation and Western blot analysis. The cells were lysed in a modified radioimmune precipitation buffer ( $1 \%$ Triton $\mathrm{X}-100,0.1 \%$ SDS, $0.1 \%$ sodium deoxycholate, $100 \mathrm{mM} \mathrm{NaCl}, 10 \mathrm{mM}$ Tris-HCl, pH 7.5, $2 \mathrm{mM}$ EDTA, $10 \mu \mathrm{g} / \mathrm{ml}$ leupeptin, $1 \mathrm{mM}$ phenylmethylsulfonyl fluoride, $10 \mathrm{mM} \mathrm{NaF}, 40 \mathrm{mM}$ B-glycerophosphate and $2 \mathrm{mM}$ $\mathrm{Na}_{3} \mathrm{VO}_{4}$ ) and insoluble material was removed by centrifugation. The protein concentration was determined by means of a Bio-Rad Protein assay (Bio-Rad, CA), and lysates containing $50 \mu \mathrm{g}$ of total cellular protein or immunoprecipitates with indicated antibodies were analyzed by Western blotting after SDS-polyacrylamide gel electrophoresis and visualized by
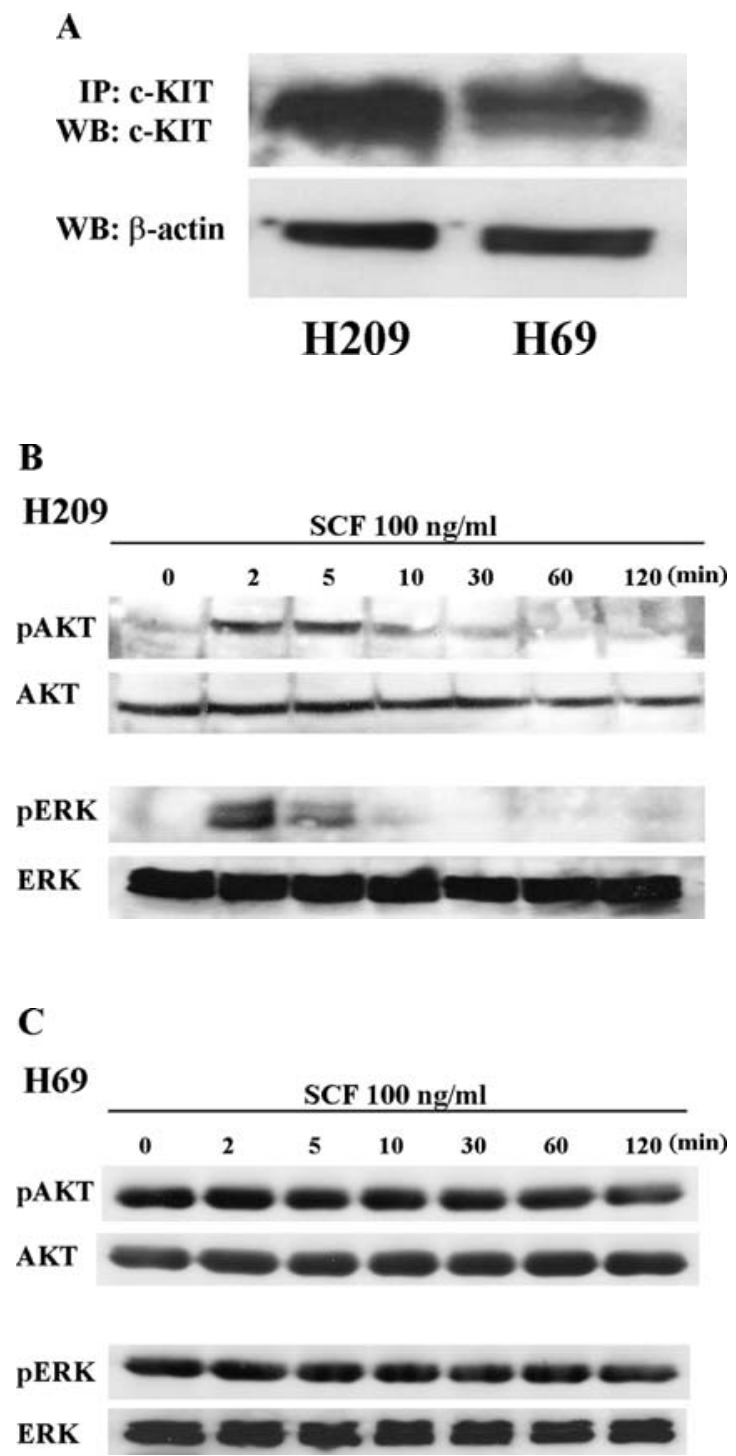

Figure 1. Effects of SCF treatment on Akt and Erk activity in H209 and H69 cells. Total cellular protein $(50 \mu \mathrm{g})$ from cell lysate was subjected to Western blot analysis with an anti-c-kit (A) as described in Materials and methods. The cells were incubated with $100 \mathrm{ng} / \mathrm{ml}$ of SCF for 2-120 min. The total cellular protein from cell lysate was subjected to Western blot analysis with anti-active Akt (pAkt) or anti-Akt (upper panel) and anti-active Erk (pErk1/2) or anti-Erk1/2 (lower panel). The results of (B) H209 cells; (C) H69 cells.

enhanced chemiluminescence detection (Amersham Pharmacia Biotech) using donkey anti-rabbit IgGs coupled to horseradish peroxidase as a secondary antibody (Amersham Pharmacia Biotech). The anti-Erk1/2 and anti-active Erk antibodies were purchased from Promega Corporation. The anti-Akt and antiactive Akt antibodies were purchased from Cell Signaling Technology. The anti-c-kit antibody was purchased from Dako Cytomation.

\section{Results}

Effects of SCF treatment on Akt and Erk activity in $\mathrm{H} 209$ and H69 cells. The effects of the SCF-treatment on Akt and Erk activation in the H209 and H69 cell lines were investigated. First, we confirmed the expression of c-kit protein in the H209 and H69 cells (Fig. 1A). Both cells were treated with $100 \mathrm{ng} / \mathrm{ml}$ 
A

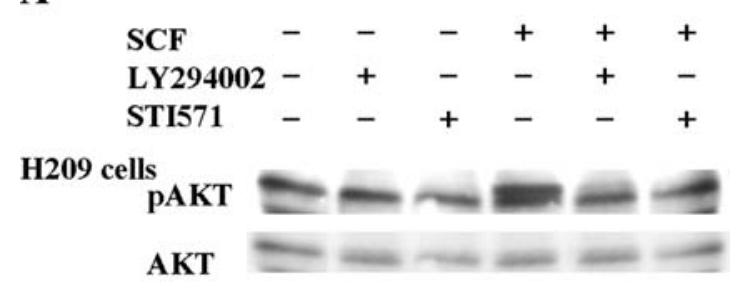

H69 cells pAKT

AKT

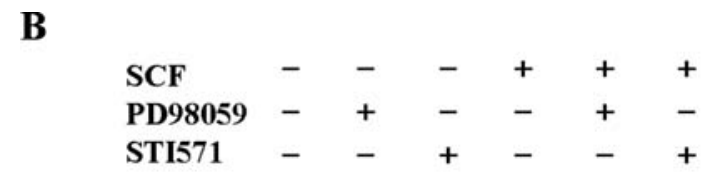

H209 cells

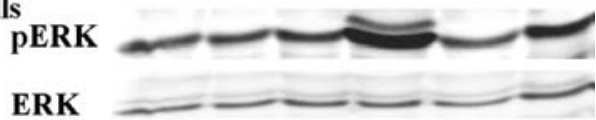

H69 cells

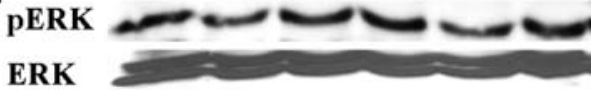

Figure 2. Effects of STI571 on the activity of Akt and Erk in H209 and H69 cells. (A) LY294002 $(25 \mu \mathrm{M})$ and $10 \mu \mathrm{M}$ of STI571 were added for $2 \mathrm{~min}$, just before the cells were incubated with $100 \mathrm{ng} / \mathrm{ml}$ of SCF. Total cellular protein $(50 \mu \mathrm{g})$ from cell lysate was subjected to Western blot analysis with anti-active Akt (pAkt) or anti-Akt as described in Materials and methods (A). In the same way, the cells were treated with $50 \mu \mathrm{M}$ of PD98059 and $10 \mu \mathrm{M}$ of STI571 for $2 \mathrm{~min}$, just before the incubation with SCF in (B). The total cellular protein from cell lysate was subjected to Western blot analysis with anti-active Erk (pErk1/2) or anti-Erk1/2 as described in Materials and methods (B)

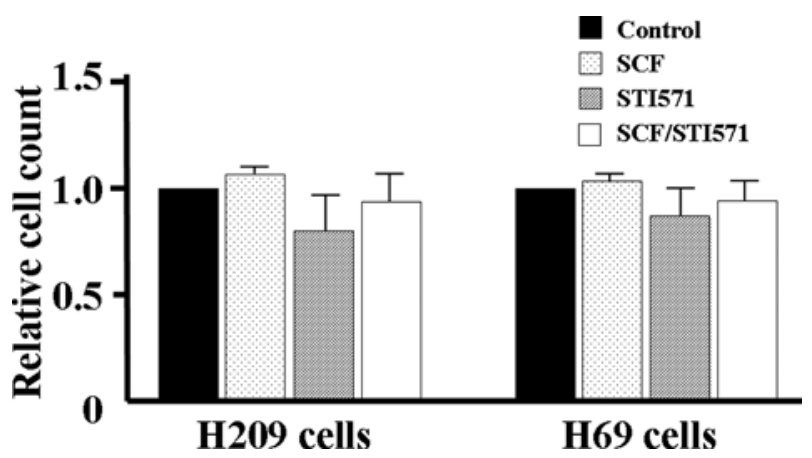

Figure 3. Effects of SCF and STI571 on the proliferation in H209 and H69 cells. Both $\mathrm{H} 209$ and $\mathrm{H} 69$ cells were treated with or without $100 \mathrm{ng} / \mathrm{ml}$ of SCF and $10 \mu \mathrm{M}$ of STI571 for $72 \mathrm{~h}$. The cell proliferation was assessed by MTT assay and expressed as the percentage of optical density (\%OD) in reference to the OD of SCF-untreated cells. Data are presented as the mean \pm standard deviation of three separate experiments.

of SCF for 0-120 min. As shown, treatment with SCF clearly activated Akt and Erk at 2-5 min in the H209 cells (Fig. 1B), but did not affect Akt and Erk activity in the H69 cells (Fig. 1C).
Effects of STI571 on the activity of Akt and Erk in H209 and H69 cells. The effect of STI571 on Akt and Erk activity was evaluated with a specific inhibitor of Akt, LY294002 or Erk, PD98059 in H209 and H69 cells.

In the absence of SCF, treatment with $25 \mu \mathrm{M}$ of LY294002 or $10 \mu \mathrm{M}$ of STI571 did not affect the activity of Akt in H209 cells. However, treatment with $25 \mu \mathrm{M}$ of LY294002 or $10 \mu \mathrm{M}$ of STI571 clearly inhibited SCF-induced Akt activation in H209 cells (Fig. 2A, upper panel). In the case of H69 cells, $25 \mu \mathrm{M}$ of LY294002 clearly inhibited the Akt activation regardless of the existence of the SCF. Ten micromolars of STI571 did not affect the activity of Akt in the H69 cells (Fig. 2A, lower panel).

Next, we investigated the effect of STI571 on the Erk activation and compared it with the effect of PD98509 on the Erk activation. In the absence of SCF, treatment with $50 \mu \mathrm{M}$ of PD98509 or $10 \mu \mathrm{M}$ of STI571 did not affect Erk activity in $\mathrm{H} 209$ cells. However, both $50 \mu \mathrm{M}$ of PD98509 and $10 \mu \mathrm{M}$ of STI571 inhibited SCF-induced Erk activation in H209 cells (Fig. 2B, upper panel). In the case of H69 cells, $50 \mu \mathrm{M}$ of PD98509 showed a subtle inhibition of Erk activity regardless of the existence of SCF. Ten micromolars of STI571 did not affect Erk activity in the H69 cells (Fig. 2B, lower panel).

Effects of SCF and STI571 on the proliferation in H209 and H69 cells. To investigate whether SCF and STI571 had any effect on the cell proliferation, H209 and H69 cells were treated with $100 \mathrm{ng} / \mathrm{ml}$ of SCF, $10 \mu \mathrm{M}$ of STI571 or a combination with the same concentration of these agents for $72 \mathrm{~h}$. Although $100 \mathrm{ng} / \mathrm{ml}$ of SCF activated Akt and Erk in H209 cells, the proliferation of $\mathrm{H} 209$ and $\mathrm{H} 69$ cells did not differ with or without SCF treatment for $72 \mathrm{~h}$ at this concentration. The growth inhibition by $10 \mu \mathrm{M}$ STI571 alone was marginal ( 20\%) compared with untreated cells in both the H209 and $\mathrm{H} 69$ cells. In the presence of $100 \mathrm{ng} / \mathrm{ml} \mathrm{SCF}$, the treatment with $10 \mu \mathrm{M}$ of STI571 did not inhibit the cell proliferation in either of the cells (Fig. 3).

Effects of SCF treatment on cytotoxic activity of chemotherapeutic agents in $\mathrm{H} 209$ and $\mathrm{H} 69$ cells. To investigate whether SCF had any effect on the cytotoxic activity of conventional chemotherapeutic agents used in clinical practice against SCLC, we examined cell growth inhibition by CDDP, VP-16, CPT-11 and AMR in the presence or absence of SCF in H209 and H69 cells. The cells were treated with various concentrations of these agents with or without $100 \mathrm{ng} / \mathrm{ml}$ of $\mathrm{SCF}$ for $72 \mathrm{~h}$. The cytotoxic activity did not differ when these cells were treated with CDDP (Fig. 4A), VP-16 (Fig. 4B) and CPT-11 (Fig. 4C). Interestingly, the cytotoxic activity of AMR decreased only when $\mathrm{H} 209$ cells were treated with AMR in the presence of SCF. On the other hand, the cytotoxic activity of AMR did not differ when H69 were treated under the same condition (Fig. 4D).

Effects of LY294002 or PD98059 on AMR-induced cytotoxicity in SCF-treated H209 and H69 cells. To investigate whether the inhibition of Akt or Erk had any effect on the AMRinduced cytotoxic activities in the presence of SCF, H209 and H69 were exposed to various concentrations of AMR with or without $25 \mu \mathrm{M}$ of LY294002 or $50 \mu \mathrm{M}$ of PD98059. SCF 
A

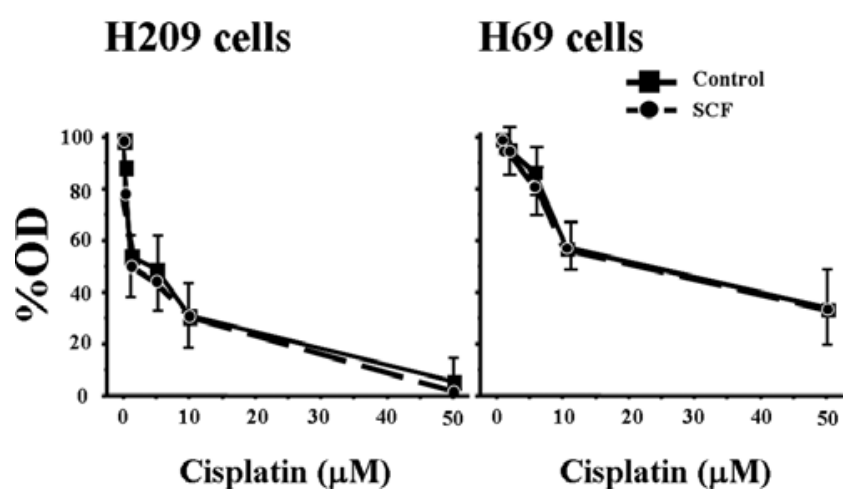

C

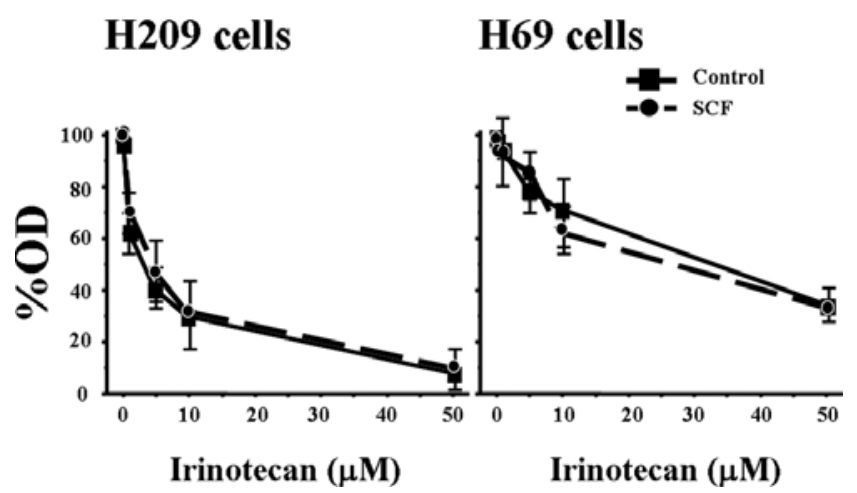

B

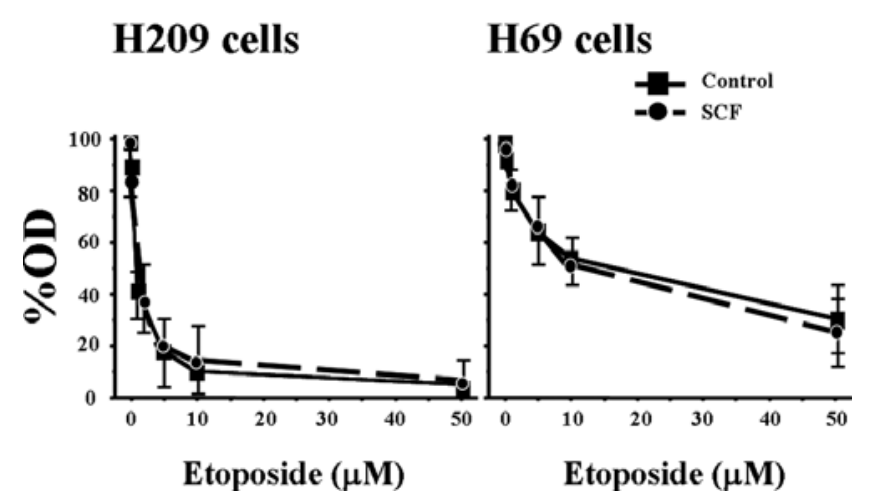

D

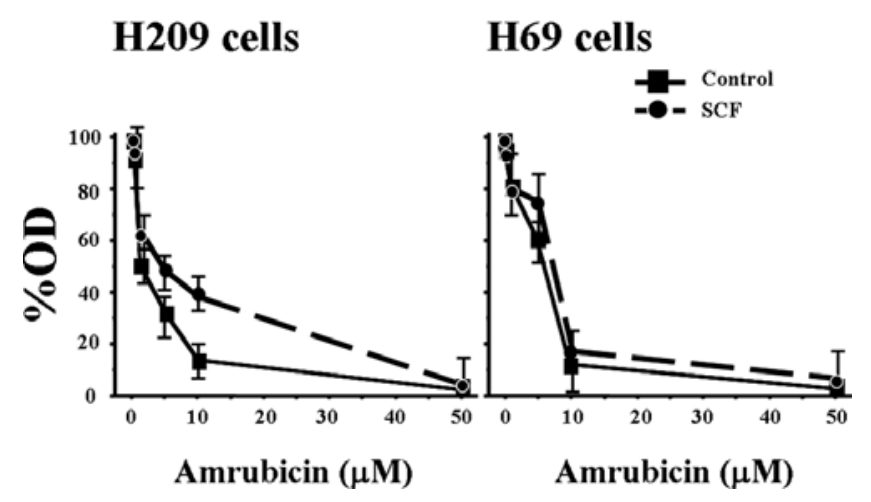

Figure 4. Effects of SCF treatment on the cytotoxic activities of cisplatin, etoposide, irinotecan and amrubicin in H209 and H69 cells. The cytotoxicity of cisplatin, etoposide, irinotecan and amrubicin was assessed by MTT assay and expressed as the percentage of optical density (\%OD) in reference to the OD of untreated cells with these chemotherapeutic agents. The results are represented on the agents used with or without SCF [(A) shows on cisplatin; (B) etoposide; (C) irinotecan and (D) amrubicin]. Data are presented as the mean \pm standard deviation of three separate experiments.

made the $\mathrm{H} 209$ cells resistant to AMR in comparison with the control and the treatment with LY294002 restored the sensitivity of $\mathrm{H} 209$ cells to the basal level. Although the sensitivity of $\mathrm{H} 69$ cells to AMR did not change in the presence of SCF, the treatment with LY294002 enhanced the sensitivity to AMR in H69 cells (Fig. 5A), whereas the treatment with PD98059 did not improve the SCF-induced AMR-resistant conditions in H209. In the H69 cells, neither SCF nor the combination of SCF and PD98059 affected the sensitivity to AMR (Fig. 5B).

Effects of SCF and STI571 on AMR-induced cytotoxicity in H209 and H69 cells. Based on these results, we examined the effects of the STI571 on the AMR-induced cytotoxicity in these two SCLC cell lines. Interestingly, when the cells were treated with $10 \mu \mathrm{M}$ of STI571, the sensitivity of $\mathrm{H} 209$ cells to AMR recovered from SCF-induced resistance to the basal level. However, neither the treatment with SCF nor the combination of SCF and STI571 had any effect on the sensitivity to AMR in H69 cells (Fig. 6).

\section{Discussion}

This in vitro study was carried out in order to investigate the possibility of SCF/c-kit-targeted therapy in the treatment of SCLC. Using c-kit protein-positive SCLC cell lines and SCF as a model system of the autocrine mechanisms, we examined the effects of STI571 on intracellular signaling, cell growth and conventional chemotherapeutic agent-induced cytotoxicity. STI571 as a single agent did not exert growth inhibition in SCF-treated SCLC cells. However, when combined with AMR, STI571 enhanced AMR induced cell growth inhibition in SCF-treated H209 cells.

In general, the binding of growth factor to its receptor activates intracellular signaling including the PI3K/Akt and Erk pathway (15). Although c-kit is detected in both H209 and H69 cells, the activation of Akt and Erk by SCF treatment was observed only in $\mathrm{H} 209$ cells. We performed all experiments in $10 \%$ FCS-containing medium. The reason that SCF did not activate Akt or Erk in H69 cells may be due to high baseline activation of these kinases by FCS. Thus, it is not surprising that the growth-promoting effect by SCF or growth-inhibitory effect by STI571 was not observed in the SCF-treated H69 cells. In addition, although SCF activated Akt and Erk, and STI571 inhibited the activation of these kinases in SCF-treated H209 cells, neither the growth-promoting effect by SCF nor the growth-inhibitory effect by STI571 was observed. H209 cells harbor loss-of-function retinoblastoma $(\mathrm{Rb})$ protein $(16)$. It was reported that $\mathrm{Rb}$ protein inactivation, which is observed in a majority of SCLC cases, makes Erk activation dispensable for the growth of SCLC (17). We speculate that the lack of growth-promoting effect by SCF and growth-inhibitory effect 
A

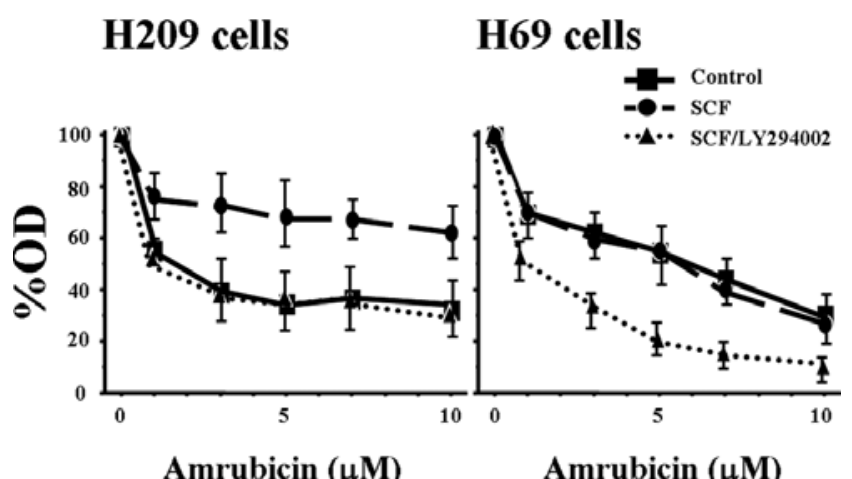

B

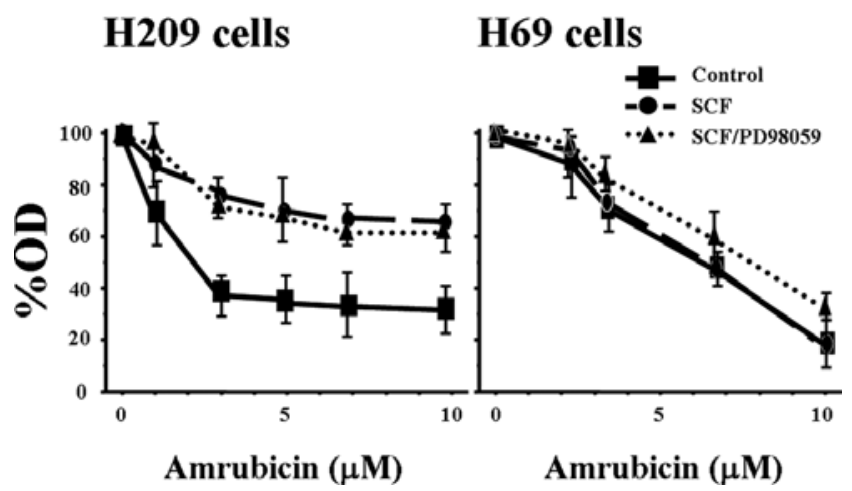

Figure 5. Effects of SCF and LY294002 or PD98059 on amrubicin-induced cytotoxicity in $\mathrm{H} 209$ and H69 cells. The cytotoxicity was assessed by MTT assay and expressed as the percentage of optical density (\%OD) in reference to the OD of amrubicin-untreated cells. The results are of agents used with SCF [(A) LY294002; (B) PD98059]. Data are presented as the mean \pm standard deviation of three separate experiments.

H209 cells

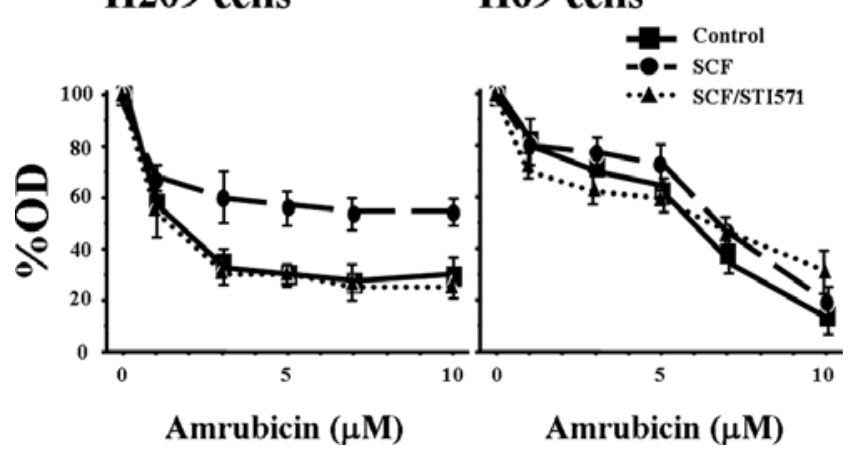

Figure 6. Effects of SCF and STI571 on amrubicin-induced cytotoxicity in H209 and H69 cells. The cytotoxicity was assessed by MTT assay and expressed as the percentage of optical density (\%OD) in reference to the OD of amrubicin-untreated cells. Data are presented as the mean \pm standard deviation of three separate experiments.

by STI571 in SCF-treated H209 cells may be attributable to functional deficiency of the $\mathrm{Rb}$ protein in the $\mathrm{H} 209$ cells.

These observations may account for the failure of the STI571-monotherapy against SCLC. Even though c-kit is expressed, STI571 may not be effective in cases where growthpromoting signals other than $\mathrm{SCF} / \mathrm{c}$-kit are dominant, as in H69 cells. Assuming SCF/c-kit mediated activation of Akt and Erk, STI571 as a single agent may not be growth-inhibiting for SCLC cells, as observed in $\mathrm{H} 209$ cells since $\mathrm{Rb}$ protein is deficient in a majority of SCLC (18).

Although STI571 did not exert cell growth inhibitory activity in SCF-treated SCLC cells, this agent inhibited SCFinduced Akt and Erk activation in $\mathrm{H} 209$ cells. It has been reported that inhibition of the PI3K/Akt pathway sensitizes SCLC cells to chemotherapeutic agents (19). Accordingly, even if STI571 is ineffective as a single agent in the treatment of SCLC, there is still a possibility that this agent may be effective when combined with a conventional chemotherapeutic agent. Based on this hypothesis, we explored the effect of SCF and STI571 on the cytotoxicity induced by several chemotherapeutic agents.

In $\mathrm{H} 69$ cells, the sensitivity to CDDP, VP-16, CPT-11 or AMR did not change regardless of the treatment with SCF. However, in $\mathrm{H} 209$ cells, treatment with SCF interfered with the cytotoxicity of AMR, but not of any other agents, suggesting that SCF-induced drug resistance may be drugspecific. Since SCF did not influence the Akt or Erk activity of $\mathrm{H} 69$ cells in our experimental condition, it is natural that the sensitivity to the chemotherapeutic agents was not affected by the treatment with SCF in H69 cells.

To clarify the mechanism by which SCF confers AMR resistance to $\mathrm{H} 209$ cells, the effects of an Akt or Erk inhibitor on AMR sensitivity were evaluated in SCF-treated cells. An Akt inhibitor, LY294002, restored SCF-induced AMR resistance to the basal level in $\mathrm{H} 209$ cells. Interestingly, although SCF did not affect AMR sensitivity in H69 cells, the treatment with LY294002 enhanced the cell growth inhibitory activity of AMR. In addition, an Erk inhibitor, PD98059, did not have any effect on SCF-induced AMR resistance in H209 cells or AMR sensitivity in H69 cells. These observations suggest that Akt activity, but not Erk activity, is strongly related with AMR sensitivity and the suppression of Akt activity sensitizes SCLC cells to AMR.

Similar to LY294002, STI571 inhibited SCF-induced Akt activation and restored SCF-induced AMR resistance in H209 cells. Therefore, recovery of AMR sensitivity to the basal level by STI571 is attributable to the inhibition of SCF-induced Akt activation.

The observation of the present study suggests that if the $\mathrm{SCF} / \mathrm{c}$-kit-mediated autocrine signal activates Akt in SCLC in vivo, STI571 may enhance the anti-tumor activity of AMR through Akt inhibition, even though STI571 as a single agent is ineffective in terms of tumor growth inhibition. Furthermore, regardless of the active $\mathrm{SCF} / \mathrm{c}$-kit-mediated autocrine loop, we propose that Akt inhibition is a promising strategy to sensitize SCLC to AMR.

In terms of combination therapy using STI571, there are two reports in which STI571 was combined with CDDP and CPT-11, or carboplatin and CPT-11 in the treatment of SCLC $(14,20)$. Neither report showed improved results by the addition of STI571 compared to the results expected with chemotherapy alone. In the present study, STI571 did not affect the cytotoxicity of either CDDP or CPT-11 in both SCF-treated H209 and H69 cells, in accordance with these reports. 
However, our observation suggests the possibility that AMR is optimal as a chemotherapeutic agent combined with STI571 in the treatment of SCLC. AMR, a totally synthetic 9-aminoanthracycline, demonstrated excellent anti-tumor activity as a single-agent or combination therapy for ED-SCLC $(6,21)$. Therefore, given that SCF/c-kit-mediated autocrine signal activates Akt in SCLC in vivo, the combination of STI571 and AMR may be promising in the treatment of SCLC.

Phosphorylated Akt was detected in $68 \%$ of the tumor specimens from SCLC patients (22), suggesting a high incidence of activated PI3K/Akt pathways in SCLC cells. Multiple neuropeptides and polypeptides other than SCF are proposed to function in SCLC (23). Therefore, it is still unknown whether SCF/c-kit is a dominant autocrine loop in SCLC and which growth factor should be addressed as a molecular target. Recently, we reported that the inhibition of c-Src leads to Akt suppression and the combination of c-Srcinhibiting agents with AMR exert synergistic activity in ckit-negative N417 SCLC cells (24), consistent with the present study. Although an Akt-suppressing agent in SCLC still remains to be established, our observation that Akt suppression enhanced AMR cytotoxicity may lead to a novel strategy in treating SCLC.

In conclusion, if $\mathrm{SCF} / \mathrm{c}$-kit contributes to Akt activation in vivo, our study proposes a novel combination treatment of STI571 and AMR against SCLC. The enhancement of AMR cytotoxicity by STI571 may be attributed to the suppression of SCF-induced Akt activation by STI571 treatments. Although Akt-suppressing agents remain to be developed, we believe that our study provides evidence supporting the future use of combination chemotherapy using Akt inhibitors and AMR against SCLC.

\section{References}

1. Huncharek M and McGarry R: A meta-analysis of the timing of chest irradiation in the combined modality treatment of limitedstage small cell lung cancer. Oncologist 9: 665-672, 2004.

2. Ihde DC: Chemotherapy of lung cancer. N Engl J Med 327: 1434-1441, 1992.

3. Green RA, Humphrey E, Close H and Patno ME: Alkylating agents in bronchogenic carcinoma. Am J Med 46: 516-525, 1969.

4. Schiller JH, Kim K, Hutson P, et al: Phase II study of topotecan in patients with extensive-stage small-cell carcinoma of the lung: an Eastern Cooperative Oncology Group Trial. J Clin Oncol 14: 2345-2352, 1996.

5. Kudoh S, Fujiwara Y, Takada Y, et al: Phase II study of irinotecan combined with cisplatin in patients with previously untreated small-cell lung cancer. West Japan Lung Cancer Group. J Clin Oncol 16: 1068-1074, 1998

6. Ohe Y, Negoro S, Matsui K, et al: Phase I-II study of amrubicin and cisplatin in previously untreated patients with extensive-stage small-cell lung cancer. Ann Oncol 16: 430-436, 2005.
7. Sundstrom S, Bremnes RM, Kaasa S, et al: Cisplatin and etoposide regimen is superior to cyclophosphamide, epirubicin, and vincristine regimen in small-cell lung cancer: results from a randomized phase III trial with 5 years' follow-up. J Clin Oncol 20: 4665-4672, 2002.

8. Hibi K, Takahashi T, Sekido Y, et al: Coexpression of the stem cell factor and the c-kit genes in small-cell lung cancer. Oncogene 6: 2291-2296, 1991

9. Reber L, Da Silva CA and Frossard N: Stem cell factor and its receptor c-Kit as targets for inflammatory diseases. Eur J Pharmacol 533: 327-340, 2006.

10. O'Brien SG, Guilhot F, Larson RA, et al: Imatinib compared with interferon and low-dose cytarabine for newly diagnosed chronic-phase chronic myeloid leukemia. N Engl J Med 348: 994-1004, 2003.

11. Schnadig ID and Blanke CD: Gastrointestinal stromal tumors: imatinib and beyond. Curr Treat Options Oncol 7: 427-437, 2006.

12. Johnson BE, Fischer T, Fischer B, et al: Phase II study of imatinib in patients with small cell lung cancer. Clin Cancer Res 9: 5880-5887, 2003.

13. Dy GK, Miller AA, Mandrekar SJ, et al: A phase II trial of imatinib (ST1571) in patients with c-kit expressing relapsed small-cell lung cancer: a CALGB and NCCTG study. Ann Oncol 16: $1811-1816,2005$

14. Johnson FM, Krug LM, Tran HT, et al: Phase I studies of imatinib mesylate combined with cisplatin and irinotecan in patients with small cell lung carcinoma. Cancer 106: 366-374, 2006.

15. Tsuruo T, Naito M, Tomida A, et al: Molecular targeting therapy of cancer: drug resistance, apoptosis and survival signal. Cancer Sci 94: 15-21, 2003.

16. Shimizu E, Zhao MR, Nakanishi H, et al: Differing effects of staurosporine and UCN-01 on RB protein phosphorylation and expression of lung cancer cell lines. Oncology 53: 494-504, 1996.

17. D'Abaco GM, Hooper S, Paterson $\mathrm{H}$ and Marshall CJ: Loss of Rb overrides the requirement for ERK activity for cell proliferation. J Cell Sci 115: 4607-4616, 2002.

18. Shimizu E, Coxon A, Otterson GA, et al: RB protein status and clinical correlation from 171 cell lines representing lung cancer, extrapulmonary small cell carcinoma, and mesothelioma. Oncogene 9: 2441-2448, 1994.

19. Krystal GW, Sulanke G and Litz J: Inhibition of phosphatidylinositol 3-kinase-Akt signaling blocks growth, promotes apoptosis, and enhances sensitivity of small cell lung cancer cells to chemotherapy. Mol Cancer Ther 1: 913-922, 2002.

20. Spigel DR, Hainsworth JD, Simons L, et al: Irinotecan, carboplatin, and imatinib in untreated extensive-stage small-cell lung cancer: a phase II trial of the Minnie Pearl Cancer Research Network. J Thorac Oncol 2: 854-861, 2007.

21. Yana T, Negoro S, Takada M, et al: Phase II study of amrubicin in previously untreated patients with extensive-disease small cell lung cancer: West Japan Thoracic Oncology Group (WJTOG) study. Invest New Drugs 25: 253-258, 2007.

22. Blackhall FH, Pintilie M, Michael M, et al: Expression and prognostic significance of kit, protein kinase $\mathrm{B}$, and mitogenactivated protein kinase in patients with small cell lung cancer. Clin Cancer Res 9: 2241-2247, 2003.

23. Fischer B, Marinov M and Arcaro A: Targeting receptor tyrosine kinase signalling in small cell lung cancer (SCLC): what have we learned so far? Cancer Treat Rev 33: 391-406, 2007.

24. Ueda Y, Igishi T, Hashimoto K, et al: Synergistic cell growth inhibition by the combination of amrubicin and Akt-suppressing tyrosine kinase inhibitors in small cell lung cancer cells: Implication of c-Src and its inhibitor. Int J Oncol 34: 689-696, 2009. 